Ann. Biol. anim. Bioch. Biophys., I971, 11 (4), 733-735.

NOTE

\title{
LA FISTULATION DU JABOT DU COQ
}

\author{
Odette IVOREC-SZYLIT 1 \\ avec la collaboration technique de Y. BonNet et L. BonNET \\ Station de Recherches avicoles, \\ Centre national de'Recherches zootechniques, $I . N \cdot R$. A., \\ 78 - Jouy-en-Josas
}

La fistulation du jabot du coq a été mise au point pour l'étude de la digestion de certains nutriments dans le premier réservoir digestif des oiseaux et suivant une technique décrite pour la fistulation du rumen des ruminants (JANET, I948).

La canule utilisée est en polyéthylène et comporte trois parties (fig. r). La partie principale (B) est composée d'un pavillon de diamètre externe de $25 \mathrm{~mm}$ et de $0,4 \mathrm{~mm}$ d'épaisseur ; le col qui le prolonge a respectivement ro $\mathrm{mm}$ de diamètre intérieur et $10 \mathrm{~mm}$ de hauteur et est muni de deux pas de vis; le premier, extérieur, sert à visser la rondelle $\mathrm{C}$ de $2 \mathrm{~mm}$ d'épaisseur, le second, intérieur, sert à visser le bouchon $\mathrm{A}$; ce bouchon est en polyéthylène plein et aussi long que le col de B, afin d'éviter que l'aliment ne séjourne ni ne fermente dans cette partie.

L'opération se fait sur des animaux à jeun après anesthésie par l'éther. L'animal est couché sur le dos et déplumé au niveau du jabot sur une surface minimum. On fait une première incision médiane d'environ $30 \mathrm{~mm}$, à l'endroit où la paroi du jabot se trouve directement sous l'épiderme. On introduit la partie B dans le jabot et on recoud successivement la paroi du jabot et la peau. On pratique une seconde incision, de dimension légèrement inférieure à celle du col de $B$, parallèle à la première, par laquelle on fait ressortir le col de la canule. De cette façon la paroi du jabot et la peau adhèrent étroitement à la canule ; on évite ainsi des suppurations ultérieures à ce niveau; la canule est ensuite maintenue par la rondelle $\mathrm{C}$.

La cicatrisation de la première incision est très rapide. On peut voir sur la figure 2 des traces de cette opération sur un animal récemment fistulé. L'opération peut se faire en une vingtaine de minutes; l'animal récupère immédiatement et se nourrit normalement dès son réveil. Il convient d'éviter de déplumer par trop le coq car les plumes protègent la canule du frottement contre les grilles de la cage quand il s'alimente et l'empêchent de piquer ses cicatrices.

Les prélèvements des contenus du jabot sont effectués à l'aide d'un tube souple relié à une trompe à vide.

(1) Adresse actuelle : Laboratoire de Recherches sur la Conservation et l'Efficacité des Aliments, C. N. R. Z., 78 - Jouy-en-Josas. 
Par rapport au tubage cette technique présente divers avantages :

- elle permet de suivre les processus de digestion au cours du temps sur le même animal sans le traumatiser par des manipulations fréquentes;

- elle permet de ne prélever que les quantités d'échantillons strictement nécessaires, en général assez faibles pour ne perturber la suite des processus digestifs que d'une façon négligeable.

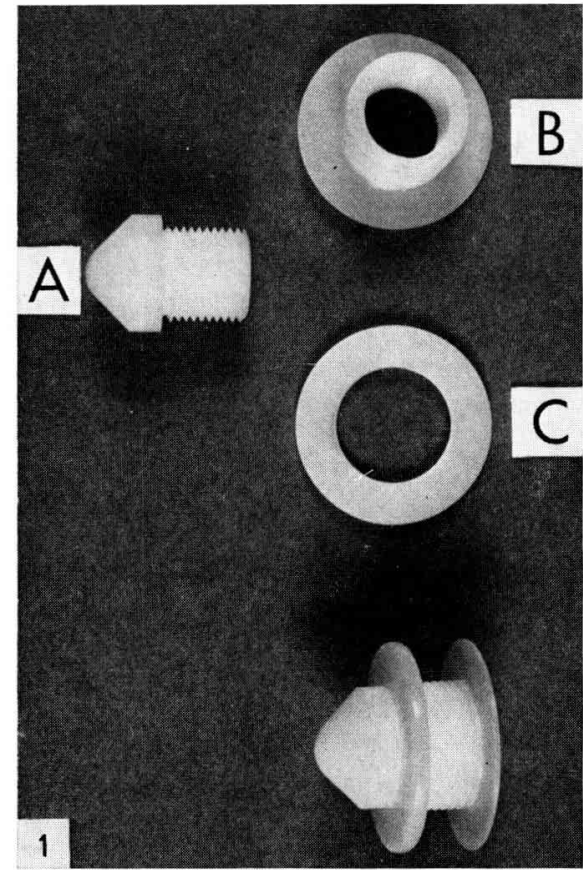

Fig. I. - Modele de canule en polyéthylène

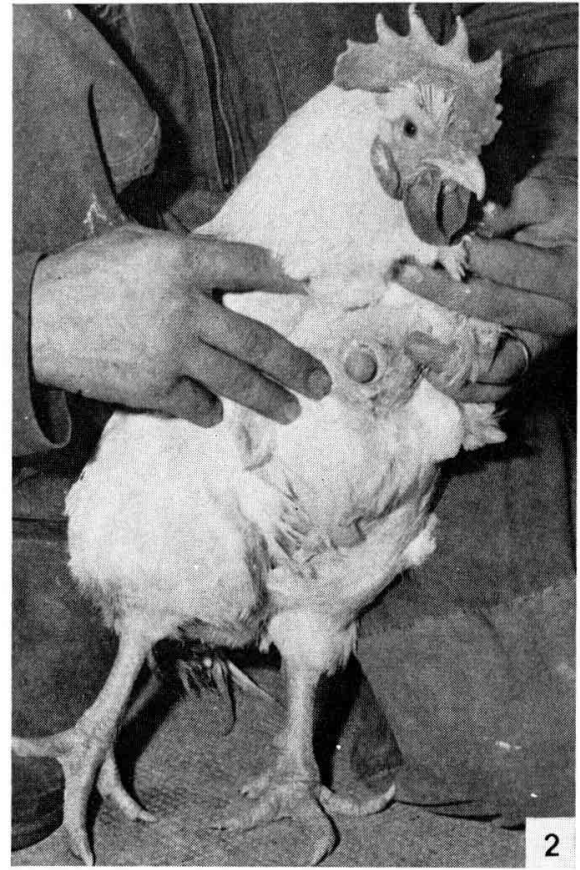

Fig. 2. - Coq porteur d'une fistule au jabot

Les plumes qui recouvrent la canule sont ici écartées. On distingue sous la canule la cicatrice de la première incision.

Cette canule se maintient en place environ 3 mois, durée suffisante pour l'étude approfondie d'un problème de digestion. Elle finit par se détacher par suite d'une kératinisation de la peau entre le pavillon et la rondelle; le détachement se fait en général d'une manière progressive et l'élimination totale de la canule a lieu au moment de la cicatrisation presque complète de la peau. Ainsi le même animal peut être éventuellement réopéré une semaine plus tard.

Rę̧u pour publication en avril 1971.

\section{SUMMARY}

\section{A TECHNIQUE FOR FISTULATING THE CROP OF THE COCK}

Crop fistulation has been performed on fasted anaesthetized cocks. The animal lying on its back is plucked over as little an area as possible.

A first median $30 \mathrm{~mm}$ incision is performed and part B of the canulla (picture $\mathrm{I}$ ) is inserted in the crop which is sutured back. 
A second button-hole incision is performed, narrow enough to allow close adherence of the skin and crop to the canulla without suturing. The canulla is then fitted to position by means of the washer $\mathrm{C}$, and closed with the stopper $\mathrm{A}$. king.

The operation is performed within 20 minutes and the animal will feed normally when awa-

Samplings are made by means of a flexible tube connected to a filter-pump.

\section{RÉFÉRENCE BIBLIOGRAPHIQUE}

JaNex I. G., I948. The production of rumen and abomosal fistulae in sheep. J. Council Sci. Ind. Res. Australia, 21, 3I1-328. 